\title{
Lesson of the month 2: Pulmonary embolism in a patient on rivaroxaban and concurrent carbamazepine
}

\author{
Authors: Thomas Burden, ${ }^{A}$ Charlotte Thompson, ${ }^{B}$ Efstathios Bonanos ${ }^{C}$ and Andrew RL Medford ${ }^{D}$
}

\begin{abstract}
A 71-year-old female with a history of pulmonary embolism treated with rivaroxaban presented with acute onset shortness of breath, chest pain and palpitations. Computed tomographic pulmonary angiography (CTPA) revealed multiple bilateral pulmonary emboli. The patient was concurrently prescribed carbamazepine and was later diagnosed with recurrence of breast cancer during the admission. We discuss common drug interactions pertinent to direct oral anticoagulants (DOACs) that can increase the risk of further venous thromboembolism. This case report highlights the importance of reviewing patient medications when considering anticoagulants and the need to raise awareness of these drug interactions among clinicians when making their choice of anticoagulation. It also reinforces the current lack of evidence for use of DOACs in patients with solid organ malignancies.
\end{abstract}

KEYWORDS: Pulmonary embolism, direct oral anticoagulants, rivaroxaban, carbamazepine, drug interactions, CYP3A4

\section{Case presentation}

A 71-year-old lady presented to the Emergency Department (ED) following 2 days of worsening breathlessness, central pleuritic chest pain and palpitations. She had been previously diagnosed with pulmonary embolism (PE) in June 2015. She was discharged on low molecular weight heparin (LMWH) due to a history of breast malignancy. Following 6 months of anticoagulation, a computed tomographic pulmonary angiogram (CTPA) excluded chronic thromboembolic disease. In December 2015, her GP commenced rivaroxaban. The GP noted the possible interaction with carbamazepine and warned the patient to observe for signs of thromboembolism.

In 2006, the patient was diagnosed with locally advanced (T4) lymph node positive right breast cancer, and treated with radical radiotherapy to the breast and lymph nodes followed by letrozole. A pulmonary nodule was noted on computerised tomography (CT)

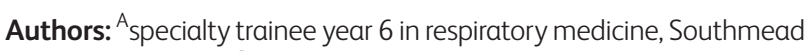
Hospital, Bristol, UK; ${ }^{\text {B }}$ foundation year 1 in respiratory medicine,

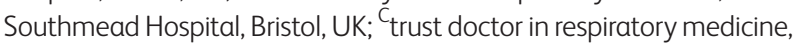

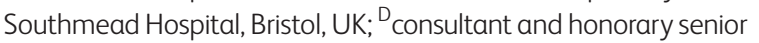
lecturer in respiratory medicine, Southmead Hospital, Bristol, UK

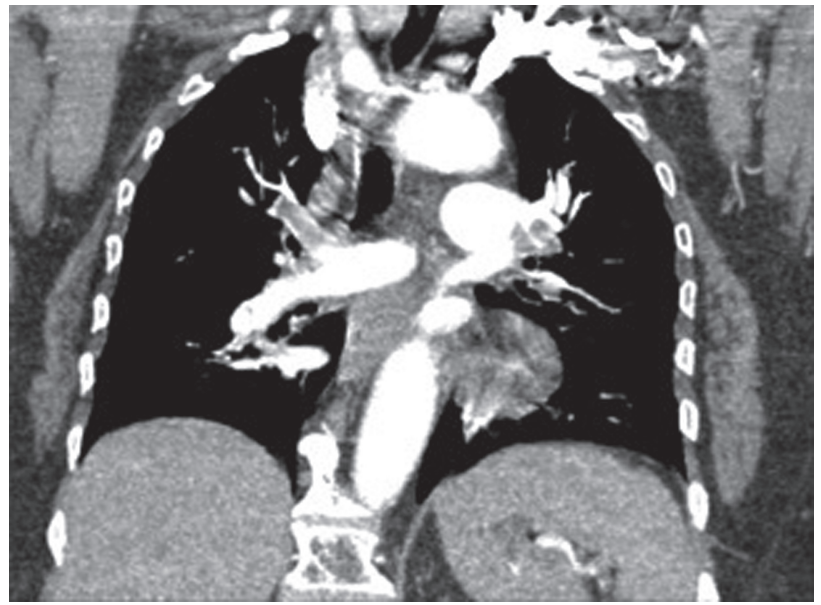

Fig 1. Coronal view of large central pulmonary emboli, contrast flowing from left subclavian vein.

during her previous admission, which was under surveillance. Her past medical history also included epilepsy, asthma, anxiety and type 2 diabetes.

Examination was significant for a tachycardia of $112 \mathrm{bpm}$, oxygen saturations $98 \%$ on $2 \mathrm{~L} /$ min supplemental oxygen, dyspnoea at rest, with a respiratory rate of 24 . There were no clinical signs of shock, right heart strain or pulmonary hypertension or of a deep venous thrombosis (DVT).

An electrocardiogram (ECG) confirmed sinus tachycardia, with $\mathrm{T}$ wave inversion in leads V1-4. Highly sensitive troponin T was $42 \mathrm{ng} / \mathrm{L}$ on admission and an age adjusted, light absorption D-dimer was positive ( $>5$ fibrinogen equivalent units). Her C-reactive protein (CRP) was raised at 51, with a mild normocytic anaemia but leucocytes were within normal parameters. A CTPA showed multiple bilateral pulmonary emboli with flattening of the intraventricular septum, suggestive of right heart strain (see Figs 1 and 2). It also identified a stable left upper lobe nodule in the lung and a right axillary lymph node, which had enlarged in size compared to previous studies.

\section{Differential diagnosis}

The combination of symptoms (acute unexplained breathlessness and chest pain with a previous malignancy and PE) made acute 


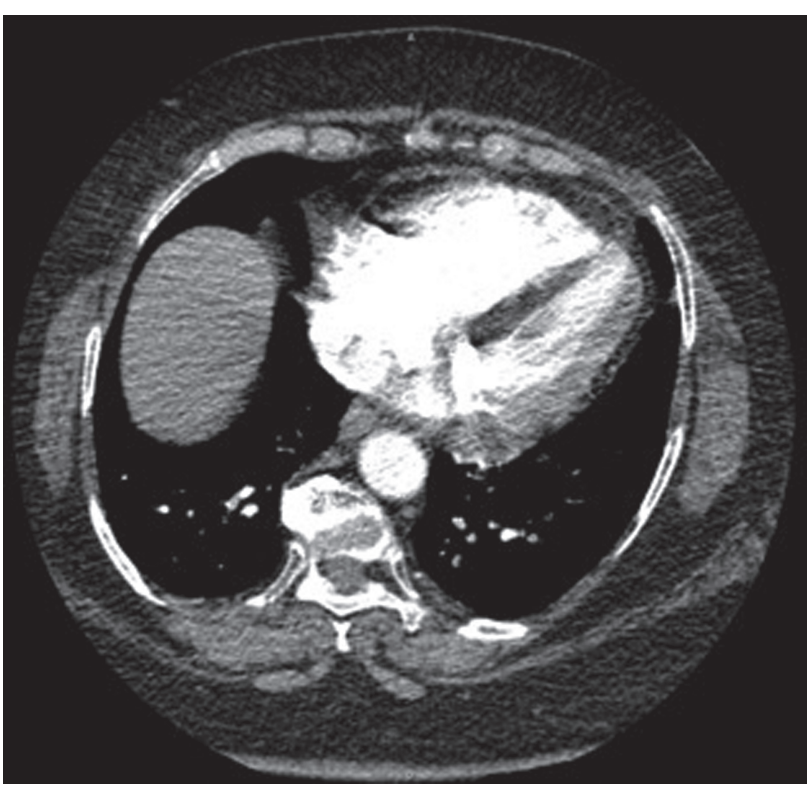

Fig 2. Axial images demonstrating intraventricular septal flattening, suggesting right ventricular strain.

PE a serious consideration. There was an absence of other clinical signs suggesting an alternative differential. The ECG demonstrated the most common finding in acute PE, sinus tachycardia, with signs consistent with right ventricular strain. Her D-dimer was elevated, although the pre-test clinical probability of PE was high and therefore D-dimer testing was not indicated as it would not have been reassuring if negative. Her troponin was raised and can occur in right heart strain from a large PE considering her ECG changes. Another consideration was the rivaroxaban, which required contemplation of a diagnosis of $\mathrm{PE}$ despite anticoagulation.

\section{Initial management and prognosis}

Monitoring occurred in a respiratory High Dependency Unit (HDU) and she was treated with therapeutic dose low-molecular-weight heparin (LMWH). Her rivaroxaban was stopped. She was referred to the rapid access breast clinic for an axillary node biopsy.

The patient reported being fully compliant with this medication, which was confirmed with checks of her dosette box. She reported taking rivaroxaban with food as advised. It was recognised that she was also on carbamazepine, which is known to reduce the efficacy of all direct oral anticoagulants (DOACs). Additionally, the patient had a suspected breast cancer recurrence, confirmed later following on node biopsy; this may also have contributed to the increased PE recurrence risk.

\section{Case progression and outcome}

The patient was discharged on lifelong LMWH due to the suspected recurrence of malignancy. An outpatient echocardiogram showed a well-functioning right ventricle with no elevated right heart pressures. Because of the potential link with her prescription, an incident form and 'yellow card' were completed.

Having confirmed nodal recurrence, the patient was followed up by the breast team and underwent breast magnetic resonance imaging (MRI). She was commenced on neoadjuvant exemestane and reviewed 2 months later for consideration of axillary surgery.

\section{Discussion}

The advent of DOACs has changed PE treatment approaches. Therefore, awareness of common drug interactions with DOACs is essential. DOACs offer a fixed dose and do not require regular monitoring. ${ }^{1}$ Their bleeding profile, when compared to warfarin, is non-inferior. ${ }^{2}$ They have well-documented side effects (eg gastrointestinal upset) and important drug interactions. Rivaroxaban is a factor Xa inhibitor. One-third is excreted in the urine and the remaining two-thirds of the drug are metabolised by the renal and hepatobiliary route involving cytochrome P450 3A4 (CYP3A4) enzymes. Rivaroxaban interacts with medications, which induce or inhibit the CYP3A4 enzymes. ${ }^{1,3}$ Inducers of CYP3A4 reduce the plasma concentration of rivaroxaban, via increased metabolism. Common CYP3A4 inducers include phenytoin, carbamazepine, phenobarbital and St John's Wort. The specific product characteristic document advises that a combination of CYP3A4 inhibitors and rivaroxaban should be avoided unless the patient is closely observed for signs and symptoms of thrombosis. All DOACs are affected by CYP3A4.

A survey of 100 medical doctors of mixed grades, including 35 registrars and 22 consultants demonstrated incomplete understanding of interactions with DOACs; $27 \%$ of responders were not aware of any interactions (see supplementary material S1) Thirteen percent were able to correctly identify all medications that interacted with DOACs. A further $28 \%$ had knowingly co-prescribed a CYP4A 4 inducer and DOACs. A comprehensive literature search showed one case report from the Netherlands on the interaction between rivaroxaban and carbamazepine. ${ }^{6}$

Warfarin prescription for venous thromboembolism (VTE) prevention has been demonstrated to be inferior to LMWH in patients with solid organ malignancies since $2003 .{ }^{4}$ To date, only phase II study evidence is available demonstrating non-inferiority of DOACs versus LMWH in solid organ malignancies and so current practice recommends $\mathrm{LMWH}$ treatment, but this may change with more evidence.

The GP documented advice to monitor closely for signs and symptoms of VTE, complying with British National Formulary guidance and Specific Product Characteristics information. There is evidence that strong CYP3A4 inducers (rifampicin) reduce circulating levels by $50 \%$. Some institutions and key opinion leaders recommend against co-prescriptions of CYP4A 4 inducers and DOACs. ${ }^{5}$ It would seem logical to recommend alternative forms of anticoagulation in this instance. The recurrence of breast cancer in this patient may have contributed to the hypercoagulable state, as this is a well-documented risk factor for VTE. Due to the recurrent breast cancer and the concurrent use of carbamazepine this patient was recommended to take life-long LMWH as per National Institute for Health and Care Excellence (NICE) guidance for anticoagulation in patients with active cancer.

\section{Key learning points}

> DOACs have many important drug interactions which need to be considered prior to prescribing.

$>$ Rivaroxaban is metabolised by CYP3A4 enzymes in the liver and serum concentration is therefore altered by CYP3A4 inducers or inhibitors 
> Awareness of these drug interactions amongst clinicians is variable

> DOACs are not currently licensed for use in malignancy, but current evidence is encouraging, treatment is still with LMWH

$>$ It is important to investigate for malignancy in patients with unexplained/recurrent venous thromboembolism.

\section{Supplementary material}

Additional supplementary material may be found in the online version of this article at www.clinmed.rcpjournal.org: S1 - online survey.

\section{Literature search terms}

A literature search was undertaken using NICE healthcare databases: AMED, EMBASE HMIC, BNI, Medline, PsycInfo, CINAHL, HEALTH BUSINESS ELITE, PUBMED and Ovid to identify studies using search terms 'pulmonary embolism', 'NOAC', 'DOAC', 'novel oral anticoagulant', 'anticoagulant', 'carbamazepine', 'anticoagulant', 'phenobarbital', 'phenytoin', 'St John's Wort' and 'Hypericum perforatum'

\section{Acknowledgements}

Written informed consent was obtained from the patient to publish the clinical details and images in this article.

\section{References}

1 McRae S. Treatment options for venous thromboembolism: lessons learnt from clinical trials. Thromb J 2014;12:27.

2 Van Es N, Coppens M, Schulman S, Middeldorp S, Büller HR. Direct oral anticoagulants compared with vitamin $\mathrm{K}$ antagonists for acute venous thromboembolism: evidence from phase III trials. Blood 2014;124:1968-75.

3 Mueck W, Kubitza D, Becka M. Co-administration of rivaroxaban with drugs that share its elimination pathways: pharmacokinetic effects in healthy subjects. Br J Clin Pharmacol 2013;76:455-66.

4 Lee AY, Levine MN, Baker RI et al. Low-molecular-weight heparin versus a coumarin for the prevention of recurrent venous thromboembolism in patients with cancer. N Engl J Med 2003;349:146-53.

5 Hansten PD, Horn JR. The top 100 drug interactions: a guide to patient management. Freeland WA: H\&H Publications, 2014.

6 Risselada AJ, Visser MJ, van Roon E. Pulmonary embolism due to interaction between rivaroxaban and carbamazepine. Ned Tijdschr Geneeskd 2013;157.

Address for correspondence: Dr Andrew RL Medford, North Bristol Lung Centre, Level 6 Gate 10, Brunel Building, Southmead Hospital, Westbury-on-Trym, Bristol BS10 5NB, UK.

Email: andrew.medford@nbt.nhs.uk

\section{Assessing trainees in the workplace An e-learning module for secondary care doctors}

Three hours of CPD-approved interactive learning covering:

$>$ feedback

$>$ supervised learning events (SLEs)

$>$ workplace-based assessments (WPBAs)

$>$ the role of the Annual Review of Competence Progression (ARCP).

For more information please visit: www.rcplondon.ac.uk/elearning

\section{Royal College} of Physicians

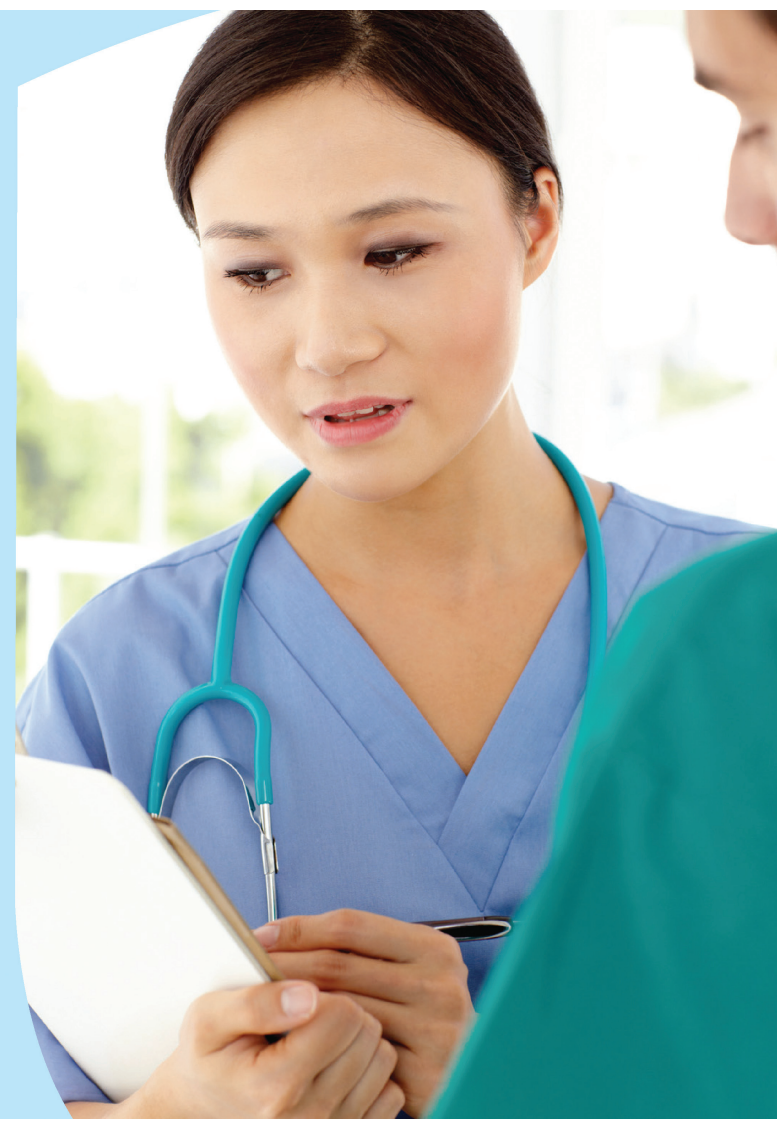

